\title{
A Case Report of Maxillary Anterior Radiolucent Lesion Associated with Impacted Teeth
}

\author{
Dr. Barsha Bajracharya, ${ }^{1}$ Dr. Pratibha Poudel, ${ }^{2}$ Dr. Dipshikha Bajracharya, ${ }^{3}$ \\ Dr. Subrata Bhattacharyya ${ }^{4}$ \\ ${ }^{1,2}$ Resident, ${ }^{3}$ Assistant Professor, ${ }^{4}$ Professor and Head, Department of Oral Pathology, \\ Kantipur Dental College, Kathmandu, Nepal
}

\begin{abstract}
The unilocular radiolucencies, with its wide range of possible diagnosis, remain the topic of much debate and interest for clinicians. These lesions cannot be diagnosed solely based on radiographic appearance, but should be based on clinical, radiological and histopathological features. Due to high probability of misdiagnosis of this entity, a sound knowledge of various unilocular radiolucencies, adequate use of diagnostic aids and careful observation is important to provide proper diagnosis and treatment. Here, we present a case of a unilocular lesion in the right anterior maxilla with two impacted teeth.
\end{abstract}

Key Words: Unilocular lesion, anterior maxilla, impacted teeth

\section{INTRODUCTION}

Unilocular radiolucencies of jaw remain a diagnostic dilemma for clinicians as many jaw lesions have similar clinical and radiographic appearance. Most of these cases are benign but can also be locally aggressive and destructive. Careful consideration of patient's history and observation of site, border and internal structure of the lesion along with its effect on the surrounding structures helps to narrow the differential diagnosis. ${ }^{1}$ In this report, we present a case of a unilocular radiolucent lesion in right anterior maxilla associated with impacted teeth.

\section{CASE REPORT}

An 18-year-old male presented with complain of retained deciduous maxillary anterior teeth. There was no history of pain or swelling in the area. He had no relevant medical or family history. Intraoral examination revealed retained 52 and 53. The adjacent gingiva and mucosa were normal. There was no swelling or tenderness in the area. An orthopantomogram (Figure1) was taken which revealed impacted 13 and

\section{Correspondence: \\ Dr. Dipshikha Bajracharya \\ Department of Oral Pathology \\ Kantipur Dental College, Kathmandu, Nepal \\ email:drdipshikhabaj@gmail.com \\ Citation \\ Bajracharya B, Poudel P, Bajracharya D, Bhattacharyya S. A case report of maxillary anterior radiolucent lesion associated with} impacted teeth. J Nep Soc Perio Oral Implantol. 2017;1:42-4. a supernumerary tooth surrounded by a well-defined radiolucency. 11 and 12 were missing. The adjacent teeth were displaced without resorption of the roots.

\section{Differential Diagnosis}

In this case, the radiographic presentation of radiolucency surrounding impacted tooth in anterior maxilla led to differential diagnosis of an odontogenic cyst or neoplasm. The first presumptive diagnosis was dentigerous cyst as it is the most common pathologic pericoronal radiolucency associated with an impacted tooth. About 0.8 to $45.9 \%$ of anterior maxillary lesions reported in English literature are odontogenic cysts. ${ }^{2}$ After the third molar, permanent maxillary canine is the most common tooth involved, which supported this differential diagnosis. ${ }^{3}$ However, unlike dentigerous cyst, the radiolucency did not show clear attachment to the cementoenamel junction.

Ranking next in the differential diagnosis was adenomatoid odontogenic tumor (AOT) due to characteristic location of

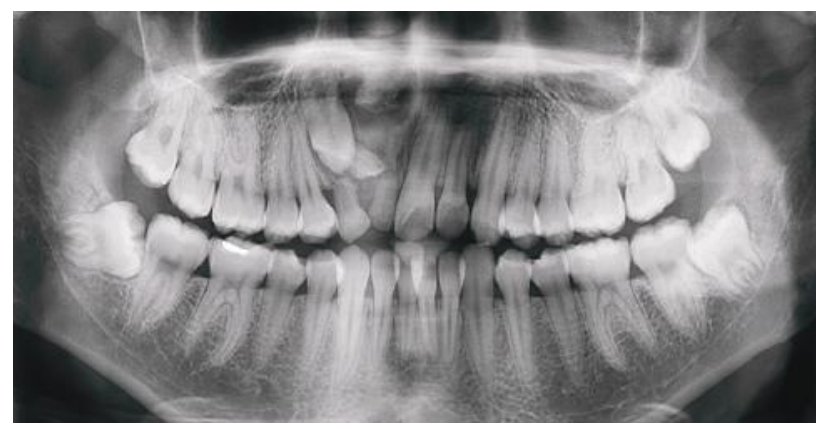

Figure 1: Preoperative Orthopantomogram showing well defined radiolucency around impacted maxillary anterior teeth 


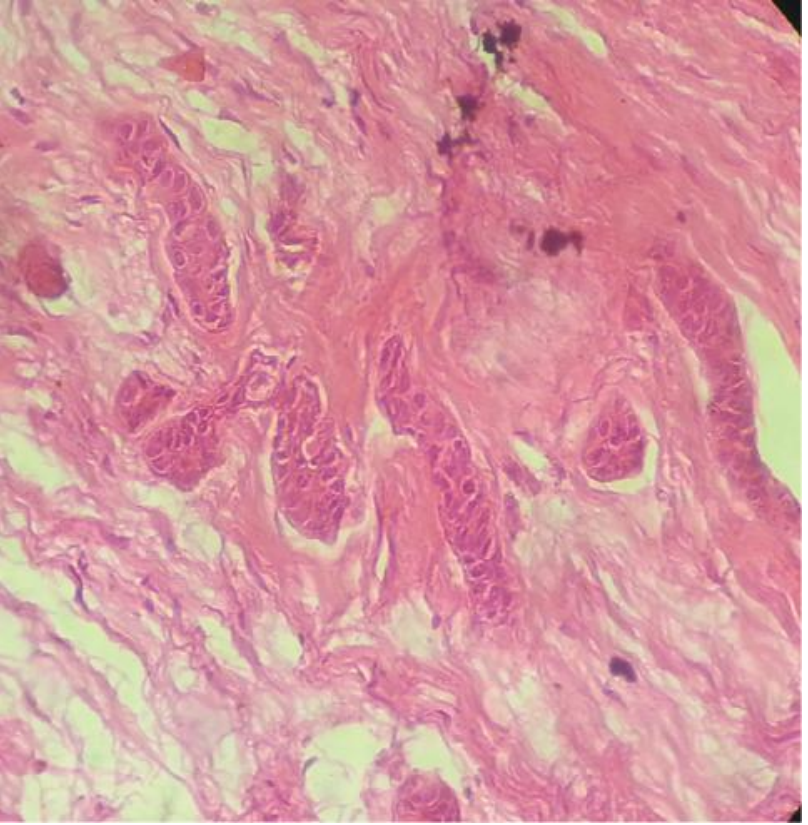

Figure 2: Strands of inactive looking odontogenic epithelium surrounded by dense bundles of collagen fibers. ( $\mathrm{H}$ and $\mathrm{E} x \mathrm{45}$ )

radiolucency in the maxillary anterior region. The occurrence of this lesion in an young patient further supported this diagnosis. ${ }^{1}$

Odontogenic myxoma was also included among the diagnostic possibilities. It mostly presents as a multilocular radiolucency, but, when they occur pericoronal to an impacted tooth, they can also present as a cyst -like unilocular lesion. ${ }^{4}$

Ameloblastic fibroma was also considered. It mostly present as an asymptomatic unilocular radiolucent lesion in the first two decades of life. However, unlike our case, the most common location is posterior mandible. ${ }^{2}$

The possibility of being a malignant tumor was considered highly improbable because of the painless asymptomatic nature, presence of a well-circumscribed radiolucency and absence of cortical destruction. ${ }^{1}$

In summary, our case involved an adolescent male with radiolucent lesion associated with impacted teeth in maxillary anterior region. The clinical and radiographic features of this case were most consistent with diagnosis of dentigerous cyst, AOT, odontogenic myxoma or ameloblastic fibroma.

\section{Diagnosis and Treatment}

In this case, complete enucleation of the lesion was performed along with extraction of the retained teeth. Histopathologic examination of the biopsy specimen showed several islands and strands of inactive looking odontogenic epithelium, composed of cuboidal cells. These islands were surrounded by dense mature connective tissue, composed of collagen fibers arranged in bundles (Figure 2). Irregular basophilic dentin and cementum like calcifications were present in

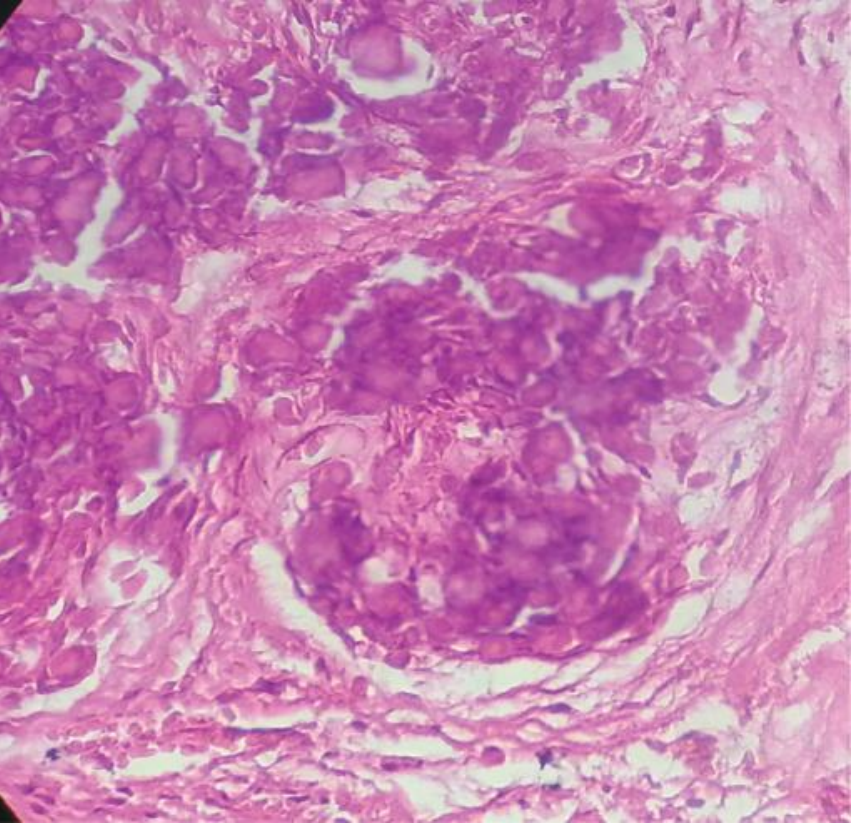

Figure 3: Irregular basophilic calcified dentin or cementum like material. (H and E x 45)

abundance (Figure 3). There was no cellular pleomorphism. Bases on these histopathological findings, final diagnosis of Central Odontogenic Fibroma (WHO Type) was made. The patient responded favourably to therapy and has shown no signs of recurrence.

\section{DISCUSSION}

Central odontogenic fibroma (COF) is a rare tumor accounting for $0.1 \%$ of all odontogenic tumors. ${ }^{5}$ Only around 70 cases of COF have been documented in English literature. It originates from odontogenic ectodermal mesenchymal tissue such as periodontal ligament, dental papilla or dental follicle. ${ }^{6}$

It mostly occurs in second to fourth decade of life and is more common in female with the ratio of 2.8 to 1.The distribution of COF between maxilla and mandible is almost equal. In maxilla, it has tendency to involve anterior area whereas in the mandible, it frequently involves posterior area. It is a slow and persistent growing benign tumor causing cortical expansion. The lesion is usually asymptomatic and can remain unknown until the appearance of a swelling. ${ }^{7}$ In our case, the lesion was asymptomatic discovered accidentally.

Radiographically, majority of COFs present as unilocular radiolucent lesion with well-defined borders while some cases appear as multilocular lesions. Few mixed radiolucent and radiopaque appearance with poorly defined borders have also been reported. COF may be associated with impacted tooth, which might give the impression of dentigerous cyst. ${ }^{5}$ This is similar to our case, where radiolucent lesion was associated with impacted canine and a supernumerary tooth. In a study by Barnes et al., 27\% of COFs were associated with 
the crown of an unerupted tooth, usually maxillary incisors followed by mandibular third molars. ${ }^{8}$

Histopathologically, the lesion is characterized by the presence of collagenous fibrous connective tissue containing varying amounts of odontogenic epithelium. Based on the amount of the odontogenic epithelium present in the lesion, WHO has defined two types of COF, the epithelium-poor type (simple type) and the epithelium-rich type (complex or WHO type). ${ }^{7}$ Epithelium-poor type of COF shows minimal cellularity with dispersed delicate collagen fibers. Fibromyxoid quality to background is given by considerable amount of ground substance. Inactive-looking odontogenic epithelium appears as irregular islands and cords. They do not show peripheral cylindrical and palisading cells or stellate reticulum-like cells. ${ }^{8}$ Occasionally, variably formed calcifications may be present. On the other hand, the epithelium-rich type of COF is composed of cellular fibroblastic connective tissue, which is similar to our case. Fibroblasts are elongated and spindle shaped. The integral component is conspicuous islands or strands of inactive-looking odontogenic epithelium, containing cuboidal cells with round or polygonal nuclei. Also, WHO type exhibits foci of calcifications of the collagenous materials which can be cementoid or dysplastic dentin. ${ }^{9}$

In general, the mode of treatment of COF is enucleation or surgical curettage with careful follow up for few years. Recurrence and malignant transformation are uncommon. ${ }^{10}$

\section{CONCLUSION}

COF is a rare benign tumor with variable clinical and radiological characteristics, which can cause a diagnostic dilemma for the clinicians. Radiographic features of COF occasionally mimic dentigerous cysts. Hence, adequate clinical, radiographical and histopathological analysis is essential in diagnosing these lesions.

\section{REFERENCES}

1. Krithika C, Vardhan BGH, Saraswathy K, Koteeswaran D, Sivapathasundaram B. Radiolucency in the anterior maxilla associated with an impacted tooth. Oral Surg Oral Med Oral Pathol Oral Radiol Endod. 2007;103(2):164-8.

2. Pinto MR, Bhandarkar G, Kini R, Naik V, Kashyap RR, Rao PK. Diagnostic Enigma of a Maxillary Unilocular Radiolucency With Multiple Impacted Teeth : A Case Report and Review of Literature. Arch Med Heal Sci. 2016;4(2):253-7.

3. Pippi R, Santoro M, Patini R. The central odontogenic fibroma : How difficult can be making a preliminary diagnosis. J Clin Exp Dent. 2016;8(2):223-5.

4. Reddy SP, Naag A, Kashyap B. Odontogenic myxoma: report of two cases. Natl J Maxillofac Surg. Medknow Publications; $2010 ; 1(2): 183$.

5. Puppala N, Madala JK, Mareddy AR, Dumpala RK. Central Odontogenic Fibroma of the Mandible. J Dent Child. 2016;83(2):94-7.

6. Soolari A, Khan A. Central Odontogenic Fibroma of the Gingiva : A Case Report. Open Dent J. 2014;8:280-8.

7. Barnes L, Eveson JW, Reichart P, Sidransky D. Pathology and Genetics of Head and Neck Tumours. WHO Classification of Tumour. 2005.

8. Barnes L. Surgical pathology of the head and neck. 3rd ed. India: Informa; 2000.

9. Veeravarmal V, Madhavan RN, Nassar MM, Amsaveni R. Central odontogenic fibroma of the maxilla. J oral Maxillofac Pathol. 2013;17(2):319.

10. Covani U, Crespi R, Perrini N, Barone A. Central odontogenic fibroma: a case report. Med Oral. 2005;10(12):154-7. 